\title{
Predictive factors of drainage volume and drain duration after the inframammary approach to nipple- areolar-complex sparing mastectomy and implant-based breast reconstruction
}

Yoon Soo Kim ${ }^{1}$, Ho Sung Kim ${ }^{1}$, Eon Ju Park ${ }^{2}$, Hyo Young Kim¹, Hong Il Kim ${ }^{1}$, Jin Hyung Park ${ }^{1}$, Chang Wan Jeon ${ }^{3}$, Hyung Suk Yi ${ }^{1}$

${ }^{1}$ Department of Plastic and Reconstructive Surgery, Kosin University College of Medicine, Busan; ${ }^{2}$ DI Plastic Surgery Clinic, Seoul; ${ }^{3}$ Department of Surgery, Kosin University College of Medicine, Busan, Korea
Background The placement of a closed suction drain is indispensable for preventing serious infections; however, closed suction drains are inevitably accompanied by increases in local infections, pain, and length of hospital stay, and delays in breast cancer treatment including postoperative chemotherapy and radiotherapy. We analyzed predictive factors of total drainage volume and duration.

Methods Among patients who were diagnosed with primary breast cancer between January 2016 and December 2017, we retrospectively analyzed those who underwent immediate implant-based breast reconstruction. Factors that could affect the total volume and duration of drainage, including lipo-prostaglandin E1 use, preoperative chemotherapy, resected breast issue weight, age, body mass index (BMI), serum white blood cell count, erythrocyte sedimentation rate, and C-reactive protein (CRP) level, were analyzed.

Results The mean volume and duration of drainage were $1,213.6 \mathrm{~mL}$ and 14.8 days respectively. BMI and CRP on postoperative day (POD) 1 were correlated with the total drainage volume. Age, BMI, and resected breast tissue weight were correlated with the drainage duration. Multiple regression analysis showed that CRP level on POD 1, age, and resected breast tissue weight significantly affected the drainage duration. Multiple regression analysis also showed that the total drainage volume was significantly affected by BMI and CRP level on POD 1.

Conclusions The factors found to affect the duration of drainage in patients undergoing implant-based breast reconstruction were CRP on POD 1, age, resected breast tissue weight, and BMI. The CRP level on POD 1 and BMI influenced the total volume of drainage.

Keywords Drainage / C-reactive protein / Breast implants / Breast neoplasms / Mastectomy
Received: Sep 12, 2020 Revised: Oct 14, 2020 Accepted: Dec 31, 2020 Correspondence: Hyung Suk Yi Department of Plastic and Reconstructive Surgery, Kosin University College of Medicine, 262 Gamcheon-ro, Seo-gu, Busan 49267, Korea

Tel: +82-51-990-6131, Fax: +82-51-990-6312, E-mail: sencha21@naver.com

Copyright @ 2021 The Korean Society for Aesthetic Plastic Surgery.

This is an Open Access article distributed under the terms of the Creative Commons Attribution Non-Commercial License (https://creativecommons.org/licenses/by-nc/4.0/) which permits unrestricted non-commercial use, distribution, and reproduction in any medium, provided the original work is properly cited. www.e-aaps.org

\section{INTRODUCTION}

According to the U.S. National Cancer Institute, breast cancer is the most common cancer among women, accounting for approximately $33 \%$ of all cancers and $20 \%$ of cancer-related deaths in women in 2019 [1]. Mastectomy is the basis for the standard treatment of breast cancer. Therefore, with the increasing incidence of breast cancer, the rates of mastectomy and breast reconstruction surgery after mastectomy are also rapidly increasing [2]. Two main 
methods for breast reconstruction are available: the use of prosthetics such as tissue expanders or silicone implants and the use of an autologous flap. When choosing a method for breast reconstruction, the size and shape of the breast, size of the tumor, severity of malignancy, and timing and method of chemotherapy and radiotherapy must be considered [3]. According to the 2018 plastic surgery statistics report from the American Society of Plastic Surgeons, $>80 \%$ of breast reconstructions performed worldwide involve the use of tissue expanders and implants [4]. One of the common complications after breast implant reconstruction is seroma, with an incidence ranging from $3 \%$ to $85 \%$ [5]. The presence of seroma is closely related to the postoperative drainage volume. Drainage fluid results from the accumulation of serous fluid in the dead space under the mastectomy skin flap, which is a natural process that occurs in the acute inflammatory response following wound healing after surgical injury [6].

To prevent the collection of fluid in the surgical area, current guidelines recommend the placement of a closed suction drain and its removal when the drainage amount becomes $<30 \mathrm{~mL} /$ day [7]. A large drainage volume is associated with a longer period of placement and a higher risk of infection. This can lead to prolongation of antibiotic treatment, restrictions in washing, extension of hospital stay, and delay of adjuvant therapy such as chemotherapy or radiation therapy. Several studies have investigated factors that can predict the amount of drainage fluid and the duration of postoperative drainage; however, the results have not been consistent $[2,8]$.

Furthermore, a study measured levels of interleukin, a major mediator of the inflammatory response, and reported that seroma formation after mastectomy is most likely a pro-inflammatory process [9]. Conversely, C-reactive protein (CRP) is an acute-phase reactant that is synthesized in hepatocytes and detected at a level of $<1 \mathrm{mg} / \mathrm{L}$ in the plasma of healthy humans. The CRP level increases within 4-6 hours after inflammation or tissue damage and thereafter rapidly decreases within 24 hours after the resolution of inflammation [10,11]. The timing of drain removal is an important factor for the return of patients to routine life after surgery. In this study, we aimed to analyze various factors that influence the total amount and duration of drainage.

\section{METHODS}

\section{Patients}

Patients who were diagnosed with primary breast cancer between January 2016 and December 2017 and underwent nipple-areolarcomplex sparing mastectomy with immediate implant-based reconstruction were included in this study. This study was approved by the Institutional Review Board of Kosin University Gospel Hospital (IRB No. 2018-04-009). Written informed consent was obtained from the patients. A retrospective chart review was con- ducted. The medical records of the patients were analyzed for age, body mass index (BMI), resected breast tissue weight, surgical information, metastasis, preoperative chemotherapy and radiotherapy, and comorbidities. A preoperative evaluation conducted by an anesthesiologist confirmed that all hematologic test values required for general anesthesia, including CRP levels, were normal. Patients with preoperative radiotherapy, metastatic breast cancer, delayed reconstruction, autologous reconstruction, uncontrolled diabetes, endocrine diseases, and smoking habits were excluded from the study.

\section{Operative methods}

An inframammary fold incision was considered first unless there was preoperative evidence that the cancer location or size would complicate an areolar incision. Single-stage reconstruction was performed in all patients who underwent nipple-sparing mastectomy. A single plastic surgeon performed the reconstruction procedures using standardized operative techniques according to the dual-plane approach. After the completion of mastectomy, the most appropriate implant was intraoperatively selected using an implant sizer to ensure breast symmetry. The lower border of the pectoralis major muscle was disinserted with Bovie electrocautery, and wounds were irrigated with cefazedone. The silicone implant used was Natrelle Style 410, which is an anatomically shaped, textured, cohesive silicone gel-filled implant (Allergan Inc., Irvine, CA, USA). Once the skin excess and flap thickness had been assessed and the surgeon had decided to perform dual-plane breast reconstruction with an acellular dermal matrix (ADM), the ADM was prepared. Implants were placed under the pectoralis major muscle, and the ADM was secured along the inferolateral pole defect using 3-0 Vicryl sutures (Ethicon, Somerville, NJ, USA). The $\mathrm{ADM}$ was meshed with a scalpel to increase pliability and facilitate fluid transfer from the pocket to the superficial layer where the drain was placed. Four types of ADM were used: Alloderm (LifeCell Corp., Branchburg, NJ, USA), Surederm (Hans Biomed Co., Seoul, Korea), Megaderm (L\&C BIO Inc., Seongnam, Korea), and Matriderm (Dr. Suwelack Skin and Healthcare Ltd., Billerbeck, Germany). The ADM product was chosen by the plastic surgeon considering the most appropriate size for covering the inferolateral pole defect. Two closed suction drains (Baro-Vac; Sewoon Medical, Cheonan, Korea) were placed: one beneath the pectoralis major muscle and the other beneath the skin flap (Fig. 1).

\section{Postoperative care}

All patients underwent basic laboratory measurements, including CRP levels, white blood cell count, and erythrocyte sedimentation rate, at the same time on postoperative days (PODs) 1,4 , and 7. The drainage volume over 24 hours was recorded each day at the same time. The closed suction drain was removed when the drainage amount became $<30 \mathrm{~mL}$ over 24 hours. An intravenous anti- 


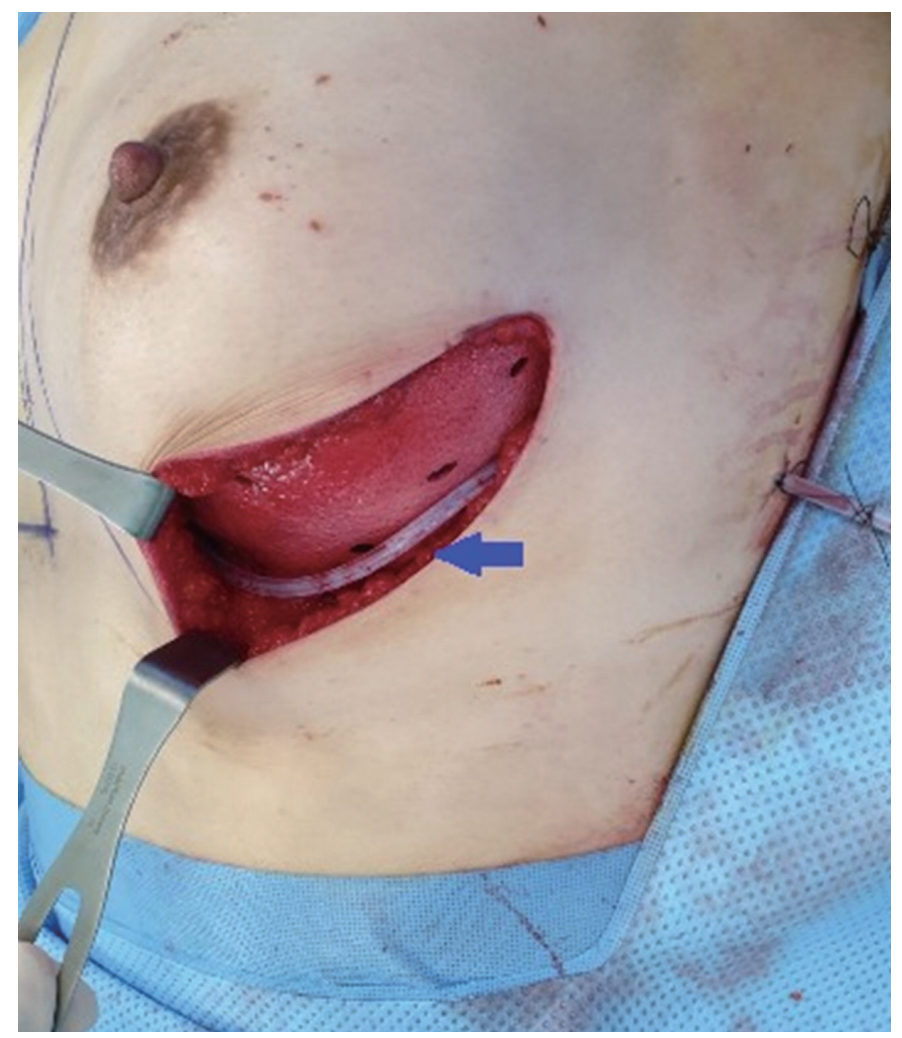

Fig. 1. Representative intraoperative photograph. A closed suction drain (blue arrow) was placed beneath the skin.

biotic (ceftazidime, $2 \mathrm{~g} /$ day) was injected until the removal of the closed suction drain. After surgery, lipo-prostaglandin E1 (Eglandin; Mitsubishi Tanabe Pharma Korea, Seoul, Korea) was used for 3 days if a decrease in the blood supply in the breast tissue was expected.

\section{Statistical analysis}

The collected data were analyzed using SPSS for Windows version 18.0 (SPSS Inc., Chicago, IL, USA), as enumerated below. In all statistical comparisons, significance was recognized as a P-value of $<0.05$.

Frequency analysis and descriptive statistical analysis were conducted for the patients' characteristics. The Mann-Whitney test was performed to verify the existence of significant differences in the duration and total volume of drainage between patients with and without lipo-prostaglandin E1 use, between patients with and without preoperative chemotherapy, and among patients with different types of ADM. Pearson correlation analysis was conducted to confirm the correlation between the main variables such as age, BMI, resected breast tissue weight, drainage duration, total drainage volume, and CRP level on POD 1. Multiple regression analysis was performed to determine the factors affecting the total drainage volume and drainage duration.
Table 1. Patient characteristics

\begin{tabular}{lc}
\hline Characteristics & Value \\
\hline Age lyr) & $47.0 \pm 8.2(25-65)$ \\
Body mass index $\left(\mathrm{kg} / \mathrm{m}^{2}\right)$ & $22.9 \pm 3.4(16.77-34.77)$ \\
Resected breast tissue weight $(\mathrm{g})$ & $294.3 \pm 203.5(45-1,424)$ \\
Duration of drainage $($ day) & $14.8 \pm 5.8(5-31)$ \\
Volume of drainage $(\mathrm{mL})$ & $1,213.6 \pm 1,053.4(140-7,965)$ \\
Type of ADM & $27(28.4)$ \\
Alloderm & $19(20.0)$ \\
Surederm & $35(36.8)$ \\
Megaderm & $14(14.7)$ \\
Matriderm & 35 \\
Lipo-prostaglandin E1 & 32 \\
Preoperative chemotherapy & \\
\hline
\end{tabular}

Values are presented as mean \pm SD (range) or number $(\%)$. ADM, acellular dermal matrix.

\section{RESULTS}

\section{Patients' characteristics}

A total of 94 female patients were included in the study. One of the included patients had bilateral breast cancer, and 95 breasts were studied in total. Table 1 shows the results of the frequency analysis and the descriptive statistics of the patients' characteristics. The average patient age was 47.0 years (standard deviation $[\mathrm{SD}]=8.2$ years), and the mean BMI was $22.9 \mathrm{~kg} / \mathrm{m}^{2}$ ( $\mathrm{SD}=3.4$ years). The weight of the resected breast tissue ranged widely, from 45 to 1,424 g. The average resected breast tissue weight was $294.3 \mathrm{~g}$, and the $\mathrm{SD}$ was very large $(\mathrm{SD}=203.5 \mathrm{~g})$. The duration of drainage ranged from 5 to 31 days, with a mean duration of 14.8 days ( $\mathrm{SD}=5.8$ days). The total volume of drainage ranged from 140 to $7,965 \mathrm{~mL}$, with a mean volume of $1,213.6 \mathrm{~mL}(\mathrm{SD}=1,053.4 \mathrm{~mL})$. The most commonly used ADM was Megaderm (35 breasts, 36.8\%), followed by Alloderm (27 breasts, 28.4\%), Surederm (19 breasts, $20.0 \%$ ), and Matriderm (14 breasts, 14.7\%). Lipo-prostaglandin E1 was used in 35 patients (36.8\%), and 32 patients (33.7\%) received preoperative chemotherapy (Table 1).

\section{Comparison of average duration and total volume of drainage according to various variables}

The Mann-Whitney test was performed to verify the existence of significant differences in the average duration and total volume of drainage between patients with and without lipo-prostaglandin E1 use, between patients with and without preoperative chemotherapy, and among patients with different types of ADM. The duration of drainage was 14.5 days in patients who used lipo-prostaglandin $\mathrm{E} 1$ and 14.7 days in those who did not, and 14.3 days in patients who received preoperative chemotherapy and 14.8 days in those 
who did not. The duration according to different types of ADM was as follows: Alloderm, 15.6 days; Surederm, 15.0 days; Megaderm, 14.3 days; and Matriderm, 16.0 days (Table 2). The average duration of drainage was not significantly different between groups according to lipo-prostaglandin E1 use, preoperative chemotherapy, and the type of ADM. The total volume of drainage was 1,075.6 $\mathrm{mL}$ in patients who used lipo-prostaglandin $\mathrm{E} 1$ and $1,227.5 \mathrm{~mL}$ in those who did not, and 1,091.8 $\mathrm{mL}$ in patients who received preoperative chemotherapy and $1,211.9 \mathrm{~mL}$ in those who did not. The volume according to different types of ADM was as follows: Alloderm, 1,168.1 mL; Surederm, 1,109.9 mL; Megaderm, 1,581.8 mL; and Matriderm, $793.3 \mathrm{~mL}$ (Table 2). As with the average duration of drainage, the average total volume of drainage was not significantly different between groups according to lipo-prostaglandin E1 use, preoperative chemotherapy, and the type of ADM.

\section{Correlation analysis}

Correlation analysis was performed to determine the coefficients of correlation between the main variables, such as age, BMI, resect-

Table 2. Mean duration and volume of drainage among the four ADM types

\begin{tabular}{lcc}
\hline ADM type & Duration of drainage (day) & Volume of drainage $(\mathrm{mL})$ \\
\hline Alloderm & 15.6 & $1,168.1$ \\
Surederm & 15.0 & $1,109.9$ \\
Megaderm & 14.3 & $1,581.8$ \\
Matriderm & 16.0 & 793.3 \\
\hline
\end{tabular}

Values are presented as mean.

ADM, acellular dermal matrix.

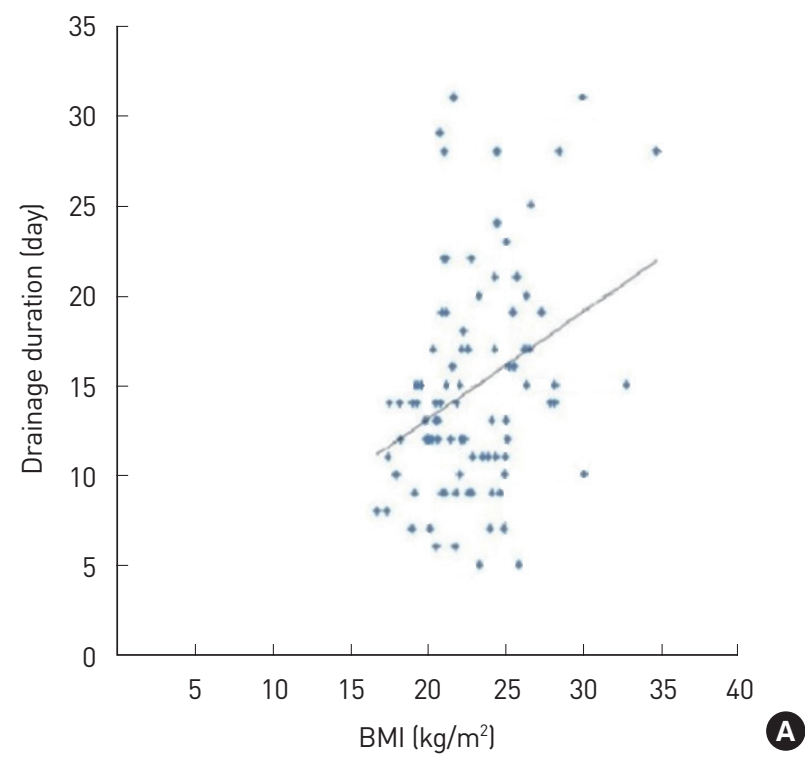

ed breast tissue weight, drainage duration, total drainage volume, and CRP level on POD 1. In this analysis, age showed a significant positive correlation with drainage duration $(\mathrm{r}=0.374, \mathrm{P}<0.05)$ (Fig. 2); BMI showed a significant positive correlation with drainage duration $(\mathrm{r}=0.256, \mathrm{P}<0.05)$ (Fig. $3 \mathrm{~A})$ and total drainage volume $(r=0.233, P<0.05)$ (Fig. 3B); resected breast tissue weight showed a significant positive correlation with drainage duration $(\mathrm{r}=0.280, \mathrm{P}<0.01)$ (Fig. 4); drainage duration showed a significant positive correlation with total drainage volume $(\mathrm{r}=0.473, \mathrm{P}<0.01)$ and CRP level on POD $1(\mathrm{r}=0.232, \mathrm{P}<0.05)$ (Fig. $5 \mathrm{~A})$; and total

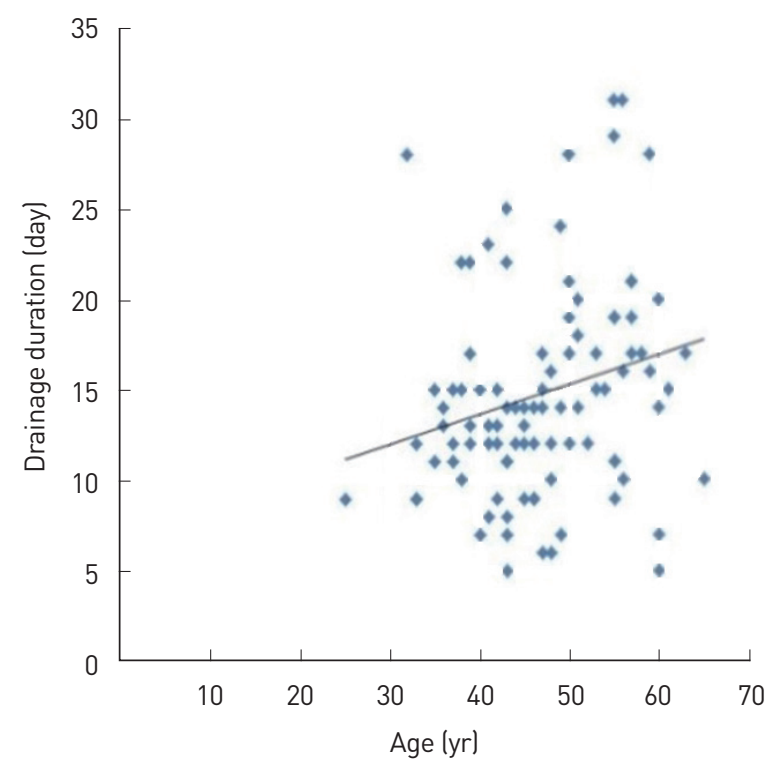

Fig. 2. Correlation between age and drainage duration. The correlation coefficient was 0.374 .

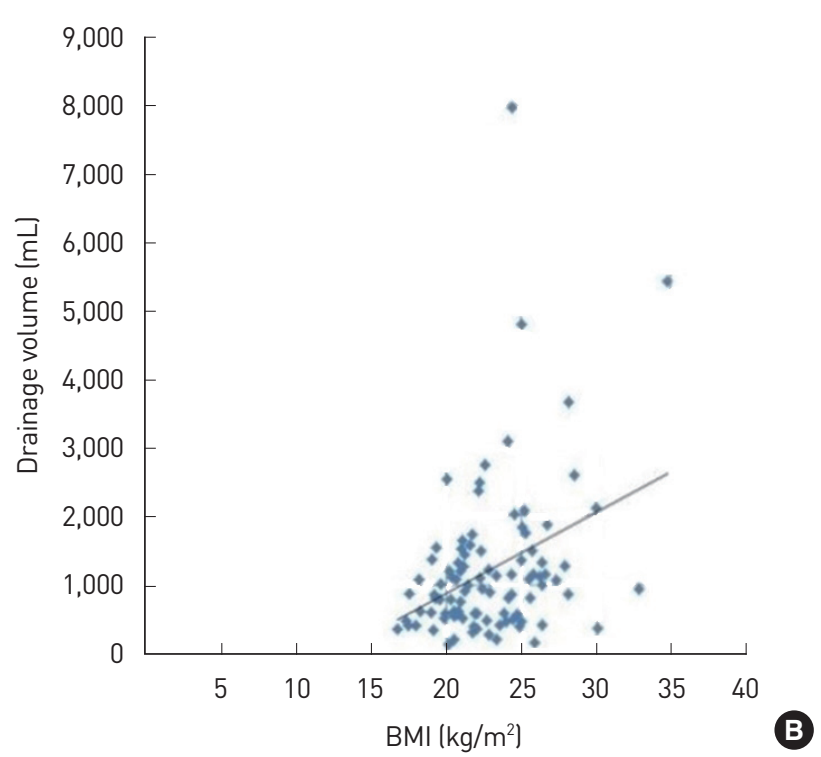

Fig. 3. Correlations between body mass index (BMI), drainage duration, and total drainage volume. (A) Correlation between BMI and drainage duration. The correlation coefficient was 0.256. (B) Correlation between BMI and total drainage volume. The correlation coefficient was 0.233 . 
drainage volume showed a significant positive correlation with CRP level on POD 1 ( $r=0.402, \mathrm{P}<0.01)$ (Fig. 5B). Conversely, age did not show a significant correlation with BMI, resected breast tissue weight, total drainage volume, or CRP level on POD 1. BMI did not show a significant correlation with resected breast tissue weight or CRP level on POD 1. Resected breast tissue weight did not show a significant correlation with total drainage volume or CRP level on POD 1 (Table 3).

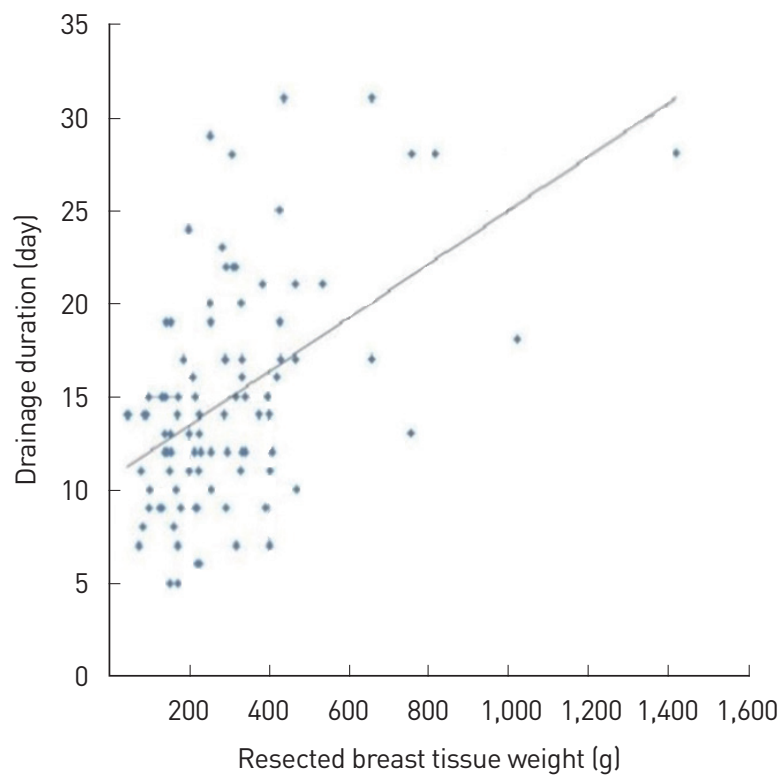

Fig. 4. Correlation between resected breast tissue weight and drainage duration. The correlation coefficient was 0.280 .

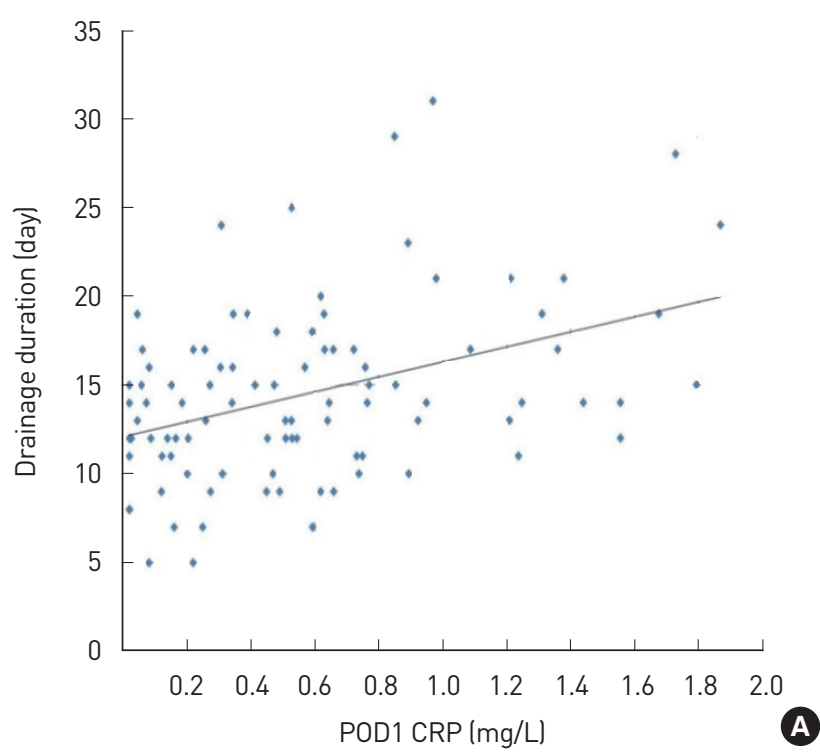

Factors influencing the average duration and total volume of drainage

Multiple regression analysis was performed to verify the effects of age, BMI, resected breast tissue weight, and CRP level on POD 1 on the average duration and total volume of drainage. Both regression models were statistically significant $(\mathrm{F}=4.503, \mathrm{P}<0.05$ and $\mathrm{F}=7.922, \mathrm{P}<0.05)$, and the explanatory power of the regression models was approximately $16.7 \%$ and $27.9 \%\left(\mathrm{R}^{2}=0.167\right.$, adj $\mathrm{R}^{2}=$ 0.130 and $\mathrm{R}^{2}=0.279$, adj $\mathrm{R} 2=0.238$ ), respectively. The Durbin-Watson statistics were 2.037 and 1.929 , respectively. As these values were close to 2, there was no problem with the assumption of independence of the residuals. Moreover, as the variance inflation factors were all $<10$, there was no problem with multicollinearity. Age $(\beta=0.277, P<0.01)$, resected breast tissue weight $(\beta=0.240$, $\mathrm{P}<0.05)$, and CRP level on POD $1(\beta=0.325, \mathrm{P}<0.01)$ had significant effects on the average duration of drainage. Furthermore, BMI $(\beta=0.166, \mathrm{P}<0.05)$ and CRP level on POD $1(\beta=0.161, \mathrm{P}<0.05)$ had significant effects on the average total volume of drainage. In other words, the average duration of drainage was found to increase with age, resected breast tissue weight, and CRP level on POD 1 , and the average total volume of drainage was observed to increase with BMI and CRP level on POD 1 (Tables 4 and 5).

\section{DISCUSSION}

Mastectomy is the foundation and the first step in the treatment of breast cancer. The serous fluids generated after breast cancer surgery are removed through closed suction drains that are inserted

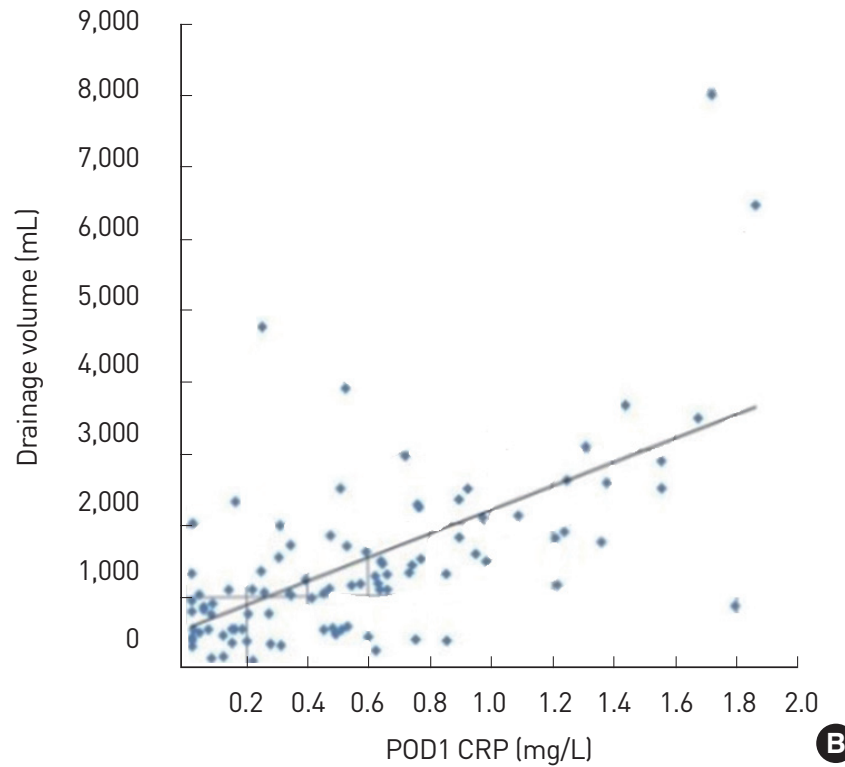

Fig. 5. Correlation between C-reactive protein (CRP) level on postoperative day (POD) 1, drainage duration, and total drainage volume. (A) Correlation between CRP level on POD 1 and drainage duration. The correlation coefficient was 0.232. (B) Correlation between CRP level on POD 1 and total drainage volume. The correlation coefficient was 0.402 . 
Table 3. Correlation analysis results

\begin{tabular}{|c|c|c|c|c|c|c|}
\hline Item & Age & BMI & $\begin{array}{l}\text { Resected breast } \\
\text { tissue weight }\end{array}$ & Drainage duration & $\begin{array}{c}\text { Total drainage } \\
\text { volume }\end{array}$ & CRP levels on POD 1 \\
\hline Age & 1 & & & & & \\
\hline $\mathrm{BMI}$ & 0.159 & 1 & & & & \\
\hline Resected breast tissue weight & -0.016 & 0.584 & 1 & & & \\
\hline Drainage duration & $0.374^{a}$ & $0.256^{\mathrm{a}}$ & $0.280^{b}$ & 1 & & \\
\hline Total drainage volume & 0.075 & $0.233^{\mathrm{a}}$ & 0.140 & $0.473^{b}$ & 1 & \\
\hline CRP levels on POD 1 & -0.044 & 0.340 & 0.233 & $0.232^{\mathrm{a}}$ & $0.402^{b}$ & 1 \\
\hline
\end{tabular}

BMI, body mass index; CRP, C-reactive protein; POD 1, postoperative day 1.

${ }^{\mathrm{a} P}<0.05 ;{ }^{\text {b }} \mathrm{P}<0.01$.

Table 4. Factors influencing the average duration of drainage

\begin{tabular}{|c|c|c|c|c|c|c|c|}
\hline Dependent variable & Independent variable & B & SE & B & $\mathrm{t}$ & P-value & VIF \\
\hline \multirow[t]{5}{*}{ Average duration of drainage } & Constant & 1.163 & 4.878 & & 0.238 & 0.812 & \\
\hline & Age & 0.196 & 0.070 & 0.277 & $2.800^{b}$ & 0.006 & 1.056 \\
\hline & $\mathrm{BMI}$ & 0.075 & 0.217 & 0.044 & 0.345 & 0.731 & 1.715 \\
\hline & Resected breast tissue weight & 0.007 & 0.003 & 0.240 & $2.004^{a}$ & 0.048 & 1.549 \\
\hline & CRP levels on POD 1 & 1.422 & 0.284 & 0.325 & $2.908^{b}$ & 0.003 & 1.044 \\
\hline
\end{tabular}

$F=4.503(P<0.05), R^{2}=0.167$, adj $R^{2}=0.130, D-W=2.037$

BMI, body mass index; CRP, C-reactive protein; POD 1, postoperative day 1; SE, standard error; VIF, variance inflation factor; D-W, Durbin-Watson.

${ }^{\mathrm{a} P}<0.05 ;{ }^{\text {b } P}<0.01$.

Table 5. Factors influencing the average total volume of drainage

\begin{tabular}{|c|c|c|c|c|c|c|c|}
\hline Dependent variable & Independent variable & B & SE & B & $\mathrm{t}$ & P-value & VIF \\
\hline \multirow[t]{5}{*}{ Average total volume of drainage } & Constant & -562.156 & 929.411 & & -0.605 & 0.547 & \\
\hline & Age & 7.174 & 13.327 & 0.056 & 0.538 & 0.592 & 1.056 \\
\hline & $\mathrm{BMI}$ & 51.864 & 41.407 & 0.166 & $1.253^{\mathrm{a}}$ & 0.021 & 1.015 \\
\hline & Resected breast tissue weight & 0.034 & 0.652 & 0.006 & 0.052 & 0.959 & 1.549 \\
\hline & CRP levels on POD 1 & 297.989 & 334.802 & 0.161 & $1.487^{\mathrm{a}}$ & 0.014 & 1.044 \\
\hline
\end{tabular}

$F=7.922(P<0.05), R^{2}=0.279$, adj $R^{2}=0.238, D-W=1.929$.

BMI, body mass index; CRP, C-reactive protein; POD 1, postoperative day 1; SE, standard error; VIF, variance inflation factor; D-W, Durbin-Watson.

${ }^{\mathrm{a}} \mathrm{P}<0.05$.

during surgery. The volume of serous fluid determines the duration of drainage, and the longer the duration, the longer the delay until the next stage of treatment [12].

An extended period of drainage increases the duration of antibiotic use, length of hospitalization, and likelihood of infection, delaying the onset of adjuvant chemotherapy [13]. Therefore, the ability to predict the drainage duration is important in clinical settings, and several studies on this issue have been published. Suga et al. [7] found that factors including age, resected breast tissue weight, and axillary lymph node dissection affected the formation of serous fluid. Theunissen et al. [2] also reported that BMI and resected breast tissue weight affected serous fluid formation [8].

In this study, various factors that may predict the amount of serous fluid were retrospectively analyzed using patients' electronic medical records. In our results, preoperative and intraoperative factors such as patient age, breast tissue resection weight, and BMI showed significant differences in terms of drainage volume or duration, as observed in previous studies.

However, the usefulness of this study lies in the advantages of CRP level on POD 1 as a predictor. Measurement of CRP levels is inexpensive and, as it involves a blood test, is easily performed after surgery. In addition, most of the previous studies analyzed patient factors (such as age and BMI) or preoperative and intraoperative factors (such as axillary lymph node dissection and resected breast size) as predictors of the occurrence of serous fluid. This makes it difficult to predict the amount of serous fluid in actual clinical settings because of the combination of decreasing and increasing trends in the data of several predictors of serous fluid. 
However, because the CRP level measured after surgery includes the effects of patient factors, intraoperative factors, and surgeonrelated factors, it most accurately represents the state of serous fluid after surgery.

This study was performed in patients with implants and ADM after mastectomy. ADM insertion after mastectomy has many advantages, such as reduction in postoperative pain, good cosmetic outcomes, and less capsular contraction. Therefore, the use of $\mathrm{ADM}$ in breast reconstruction is gradually increasing $[14,15]$. However, no studies have been published on the predictors of serous fluid in patients with an ADM inserted during mastectomy and reconstruction. This study is the first to identify the predictors of serous fluid in patients with ADM.

In addition, lipo-prostaglandin E1 is frequently administered to increase the blood flow of the flap through its effects of inducing microvascular dilation and angiogenesis. These pharmacological actions leading to increased blood flow raise the concern that lipoprostaglandin E1 may increase the amount of serous fluid, resulting in an increased amount of effusion. However, our study showed no significant relationship between the administration of lipo-prostaglandin E1 and the amount of serous fluid formed. Further follow-up studies are needed to investigate the effect of microvascular dilation induced by lipo-prostaglandin E1 and its influence on seroma formation. Therefore, lipo-prostaglandin E1 can be considered a safe medication when the blood flow to the breast flap remaining after mastectomy is poor.

The mechanism of the generation of serous fluid is not fully understood. However, the results of various published studies suggest that serous fluid formation may be due to acute inflammatory reactions in the process of surgical wound healing [16]. On the basis of these previous studies, we estimated the amount of serous fluid generated by inflammation by measuring the CRP level, which proportionally increases with increasing degree of inflammation, and obtained a suitable result. A high CRP level is a predisposing factor for seroma fluid formation, and a follow-up study is needed to compare the amount of drainage with daily follow-up measurements of CRP levels, under the assumption that even the same operation could lead to a different inflammatory response.

This study included patients who received implants after undergoing mastectomy. Our results showed that CRP levels can be useful for the quantitative prediction of serous fluid in patients who undergo mastectomy with implant-based reconstruction. However, to demonstrate the usefulness of these factors in mastectomy, further studies are required in patients undergoing simple mastectomy and those undergoing reconstructive surgery with autologous tissue and flaps after mastectomy.

The factors that affected drainage duration in our patients who underwent nipple-areolar-complex sparing mastectomy and immediate breast reconstruction with ADM and implants were CRP level on POD 1, age, and resected breast tissue weight. The factors that affected drainage volume in these patients were BMI and CRP on POD 1.

Therefore, plastic surgeons performing implant-based breast reconstruction can estimate the duration and total volume of drainage by measuring the CRP level on POD 1. Consequently, it would be possible to inform patients in advance that the drainage duration may be extended if they have high CRP levels on POD 1, thus enabling the development of more specific treatment and discharge plans.

\section{NOTES}

\section{Conflict of interest}

No potential conflict of interest relevant to this article was reported.

\section{Ethical approval}

The study was approved by the Institutional Review Board of Kosin University Gospel Hospital (IRB No. 2018-04-009) and performed in accordance with the principles of the Declaration of Helsinki.

\section{Patient consent}

The patients provided written informed consent for the publication and the use of their images.

\section{ORCID}

Yoon Soo Kim

Ho Sung Kim

Eon Ju Park

Hyo Young Kim

Hong Il Kim

Jin Hyung Park

Chang Wan Jeon

Hyung Suk Yi

\section{REFERENCES}

1. Jemal A, Murray T, Samuels A, et al. Cancer statistics, 2003. CA Cancer J Clin 2003;53:5-26.

2. Theunissen D, Cant PJ, Dent DM. Factors that influence volume and duration of wound drainage after mastectomy and level III axillary node clearance. Breast 2001;10:538-9.

3. Bang S. Breast reconstruction. J Korean Med Assoc 2011;54:10-1.

4. American Society of Plastic Surgeons. 2018 Plastic surgery statistics [Internet]. Arlington Heights, IL: The Society; c2018 [cited 2021 Jun 11]. Available from: https://www.plasticsurgery.org/news/plastic-surgery-statistics.

5. Srivastava V, Basu S, Shukla VK. Seroma formation after breast cancer surgery: what we have learned in the last two decades. J Breast Cancer 


\section{2;15:373-80.}

6. Phillips BT, Wang ED, Mirrer J, et al. Current practice among plastic surgeons of antibiotic prophylaxis and closed-suction drains in breast reconstruction: experience, evidence, and implications for postoperative care. Ann Plast Surg 2011;66:460-5.

7. Suga $\mathrm{H}$, Shiraishi T, Shibasaki Y, et al. Predictive factors for drainage volume after expander-based breast reconstruction. Plast Reconstr Surg Glob Open 2016;4:e727.

8. Talha A, Ramadan R, Abdelhamid S, et al. Postmastectomy seroma: how much is it affected by serum levels of IL- 6 and CRP and how much is it reduced by intravenous hydrocortisone injection? Egypt J Surg 2015;34:17.

9. Szecsi PB, Larsen J, Horby J, et al. Seroma production after breast cancer surgery has a pro-inflammatory component. Open Breast Cancer J 2012;4:11-7.

10. Akashi M, Furudoi S, Hashikawa K, et al. Postoperative abnormal response of C-reactive protein as an indicator for infectious complications after oral oncologic surgery with primary reconstruction. J Oto- laryngol Head Neck Surg 2015;44:13.

11. Houten JK, Tandon A. Comparison of postoperative values for C-reactive protein in minimally invasive and open lumbar spinal fusion surgery. Surg Neurol Int 2011;2:94.

12. Kweon SS. Updates on cancer epidemiology in Korea, 2018. Chonnam Med J 2018;54:90-100.

13. Kumar S, Lal B, Misra MC. Post-mastectomy seroma: a new look into the aetiology of an old problem. J R Coll Surg Edinb 1995;40:292-4.

14. Salzberg CA, Ashikari AY, Koch RM, et al. An 8-year experience of direct-to-implant immediate breast reconstruction using human acellular dermal matrix (AlloDerm). Plast Reconstr Surg 2011;127:514-24.

15. Chun YS, Verma K, Rosen H, et al. Implant-based breast reconstruction using acellular dermal matrix and the risk of postoperative complications. Plast Reconstr Surg 2010;125:429-36.

16. Aitken DR, Hunsaker R, James AG. Prevention of seromas following mastectomy and axillary dissection. Surg Gynecol Obstet 1984;158: 327-30. 